\title{
Research on the Upgrade of Evaluation System Design of Vegetables Safety of Shenzhen
}

\author{
Kunya Wu, Shuaishuai Hu \\ College of Mathematics and Physics, BOHAI University, Jinzhou, 121000, China
}

Keywords: Risk evaluation, Hierarchy analysis, Cluster analysis, Linear regression model.

\begin{abstract}
Food safety issues are national security issues. Any food safety event they not only brings people great harm, but also hurts the credibility of the government. At present, many kinds of fresh vegetables are being imported into consumer market of Shenzhen via many channels. Different habitats and production management patterns pose a new challenge to the traditional vegetable safety supervision. This paper improves the traditional method of vegetable safety supervision through modeling and analysis of the factors directly related to vegetable safety.
\end{abstract}

\section{Introduction}

At present, the population of Shenzhen is still increasing, and many kinds of fresh vegetables are being imported into the consumer market by means of many channels. However, the vegetable safety supervision work method of difference in origin and different mode of production management and other factors on the formation of the tradition of new challenges, so in view of vegetable safety supervision work to improve the traditional method. The paper uses the data of the qualified rate in the annex from January of 2016 to March of 2017in Shenzhen city, fresh vegetable samples, use the analysis method of time series prediction of Shenzhen fresh vegetables overall pass rate, and set up a linear regression model using the data and relevant data on the question of the fitting using SPSS and SAS software. To determine the main influencing factors of Shenzhen city, fresh vegetables pass rate accordingly, determine the main analysis sampling inspection plan, and according to the proposal to establish the model of reasonable sampling, the sampling model of this paper simply from the statistics of simple random sampling and stratified sampling were considered, due to the need to consider the rationality of the model in practical application to reduce the error, this paper uses statistical probability principle, unbiased estimation, in order to test the model, this paper depicts the sample variance The image has reached the end of the proposed program.

\section{Analysis of Safety Risk Factors of Fresh Vegetables of Shenzhen}

Safety Risk Factors of Fresh Vegetables of China.In this paper, the data of fresh vegetables and their unqualified samples were screened out by cluster analysis, and the main risk items of fresh vegetables were collected in the past two years. 
Table 1. Main risk items of fresh vegetables in the past two years in China

\begin{tabular}{|c|c|l|}
\hline Number & Risk items & \multicolumn{1}{|c|}{ Concrete risk items } \\
\hline \multirow{2}{*}{$\mathbf{1}$} & Pesticide residue & $\begin{array}{l}\text { Methamidophos, Omethoate, phorate, parathion, } \\
\text { methyl parathion, 50 kinds of pesticide isofenphos } \\
\text { methyl, Isocarbophos, dimethoate, dichlorvos, } \\
\text { acephate, chlorpyrifos, Triazophos three, } \\
\text { profenofos, fenitrothion, two imidacloprid } \\
\text { residues }\end{array}$ \\
\hline $\mathbf{2}$ & Food additives & $\begin{array}{l}\text { Sodium cyclamate, sulfite, saccharin sodium, } \\
\text { benzoic acid and sodium salt }\end{array}$ \\
\hline $\mathbf{3}$ & Illegal add & $\begin{array}{l}\text { Sulfur dioxide, 6- benzyl adenine (6-BA), lemon } \\
\text { yellow, blue and fluorescent material }\end{array}$ \\
\hline $\mathbf{4}$ & Contaminants & Total mercury, lead, total arsenic, total mercury, \\
& chromium, total mercury, nitrite, lead \\
\hline $\mathbf{5}$ & Bicroorganism & Coli, coliform bacteria \\
\hline $\mathbf{6}$ & Quality index & Ochratoxin A \\
\hline
\end{tabular}

In view of the above data, this paper screened out the unqualified items and their proportion in the total unqualified items of the fresh vegetables in recent two years, and carried on the visual analysis. The results are shown in the following table:

Table 2. Proportion of nonconforming items of vegetable safety of Xinxiang in recent two years

\begin{tabular}{|l|l|}
\hline \multicolumn{2}{|l|}{ Proportion of nonconforming items of vegetable safety of Xinxiang in recent two years } \\
\hline Biological toxin & $<1 \%$ \\
\hline Quality index & $<1 \%$ \\
\hline Microorganism & $<1 \%$ \\
\hline Illegal add & $5 \%$ \\
\hline Contaminants & $11 \%$ \\
\hline Food additives & $23 \%$ \\
\hline Pesticide residue & $60 \%$ \\
\hline
\end{tabular}

Based on this table, we can get the following conclusions: pesticide residues and food additives are the main factors causing fresh vegetable safety risk, which is the main cause of pesticide residues in fresh vegetables sampling unqualified. Biological toxins and quality indicators are less than 1\%, illegal additions, microbial contamination, pollutants and so on also accounted for a small proportion, that is, in fresh vegetables food safety risk inspection, these factors have a minor impact. It is basically consistent with the above analytic hierarchy process.

Safety Risk of Fresh Vegetables of Shenzhen. By the above factors of pesticide residues in food safety risk of the proportion of fresh vegetables in up to $60 \%$, thus this paper assumes that the pesticide residues are the main factors leading to the city of Shenzhen fresh vegetables are unqualified, main factors affecting fresh vegetables and food safety risk, i.e.. In order to verify the idea of this 
paper, for the Shenzhen market and quality supervision and Management Committee official website based on the data, the data integration and graphic analysis of Shenzhen city from July of 2016 to February of 2017 sampling results of vegetables, as shown in the following table.

Table 3. Data integration and graphic analysis of Shenzhen city

\begin{tabular}{|l|l|c|c|c|}
\hline Sample name & \multicolumn{1}{|c|}{$\begin{array}{c}\text { Nonconforming } \\
\text { project }\end{array}$} & $\begin{array}{c}\text { Judgment } \\
\text { requirement }\end{array}$ & $\begin{array}{c}\text { Inspection item } \\
\text { determination }\end{array}$ & $\begin{array}{c}\text { Occurrence } \\
\text { frequency }\end{array}$ \\
\hline Cowpea & Budweiser 1.3 & $\begin{array}{c}\leq 0.02 \\
\leq 0.05 \\
\leq 1\end{array}$ & Unqualified & 7 \\
\hline Spinach & $\begin{array}{l}\text { Methamidophos } \\
0.14 \\
\text { acephate } 5.08\end{array}$ & $\leq 0.1$ & Unqualified & 3 \\
\hline Celery & Chlorpyrifos 3.15 & $\leq 0.01$ & Unqualified & 3 \\
\hline Shanghai blue & Phorate 0.12 & $\leq 1$ & Unqualified & 18 \\
\hline $\begin{array}{l}\text { A variety of } \\
\text { Chinese } \\
\text { cabbage }\end{array}$ & Acetamiprid 2.12 & $\leq 0.02$ & Unqualified & 18 \\
\hline Cabbage heart & Fipronil 0.12 & $\leq 0.02$ & Unqualified & 1 \\
\hline Ternip & Omethoate 2.16 & $\leq 0.01$ & Unqualified & 1 \\
\hline $\begin{array}{l}\text { Chinese } \\
\text { Cabbage }\end{array}$ & Phorate 0.084 & $\leq 0.01$ & Unqualified & 5 \\
\hline $\begin{array}{l}\text { Chinese } \\
\text { Cabbage }\end{array}$ & $\begin{array}{l}\text { Methyl } \\
\text { phosphorous } 0.54\end{array}$ & $\leq 0.02$ & Unqualified & 2 \\
\hline
\end{tabular}

The above is the main part of the overall data analysis this paper selected, according to the analysis of vegetable monitoring project provided to annex the whole data of Shenzhen city from July of 2016 to February of 2017, fresh vegetables all sampling unqualified items are mainly belongs to the pesticide residues by comparison on the table, it can be inferred that the main reason of fresh sample Shenzhen city is unqualified vegetable pesticide residues, and the influence of the above factors make the food safety risk of vegetables in Shenzhen city is basically the same.

The above data analysis, Shenzhen City fresh vegetables food safety risk in general, pesticide residues are the dominant factors affecting the food safety of fresh vegetables, vegetables for different types and different regions, different time of the size of the risk will differ, the relevant departments to formulate plans, flexible and specific implementation plan this paper will ask detailed statement in fourth chapter.

\section{Scientific Design of Sampling Inspection Plan of Fresh Vegetables of Shenzhen}

Model Prepartion.Assuming that the total amount of fresh vegetable food is $\mathrm{N}$, the fresh vegetables and vegetables are divided into m layers according to the eight categories of appeal, and then the factors affecting the safety of fresh vegetables and foods are divided into i layers;

In this paper, the stratified sampling process is divided into two stages: sampling of eight major fresh vegetable foods, and assuming that the mathematical expectation of the unit index of the overall layer is the variance. The quantity of vegetables on the first floor is the same. Then stratified sampling, the number of samples drawn for the overall level of the first unit index value, indicating the sample layer of the first unit index value.

Model Establishment and Solving. Because species, vegetable food batches are edible from production and processing to sales, intermediate complex, quality inspection department can't do 
comprehensive quality tests for all vegetable food, usually do some sampling. For this reason, a reasonable stratified sampling model is established. By calculating the population variance and sample variance, the relative accuracy is obtained and the overall situation is estimated by some samples. We have:

$$
\begin{aligned}
& \mu_{m}=\frac{1}{N_{m}} \sum_{i=1}^{N_{m}} X_{m i} \\
& \sigma_{m}^{2}=\frac{1}{N_{m}-1} \sum_{i=1}^{N_{m}}\left(X_{m i}-\mu_{i}\right)^{2} \\
& E\left(S_{m}^{2}\right)=\sigma_{m}^{2} \\
& E\left(S_{m}^{2}\right)=E\left[\frac{1}{n_{m}}\left(X_{m i}-\overline{X_{m}}\right)^{2}\right]
\end{aligned}
$$

Unbiased estimate is the expected value of the sample estimate of the parameter, equal to the real value of the parameter. The mathematical expectation of the estimator is equal to the estimated parameter, which is called unbiased estimation. Here is the application of the progressive principle, which makes the parameter estimation value closer to the estimated parameter, and minimizes the system error.

According to the principle of variance analysis, the overall variance can be divided into two parts after stratified. One is the variance within the layer, and the other is the inter layer variance. In stratified sampling, the variance of stratified sampling is only related to the inner layer due to the independence between the layers. Therefore, reducing the variance in the layer can reduce the population variance in the stratified process, thus improving the sampling efficiency and quality of vegetable food.

Results and Analysis. According to the function of $S_{m}^{2}=\frac{1}{n_{m}}\left(X_{m i}-\overline{X_{m}}\right)^{2}$, we have:

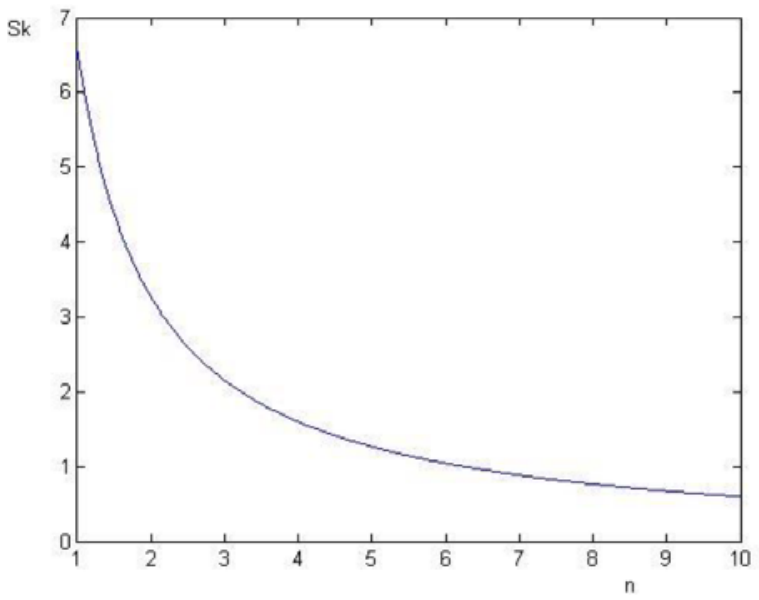

According to the image, the larger the number of samples, the smaller the sample variance, the more variance, the results of stratified sampling is more stable, closer to the true value, and can improve the sampling efficiency and quality. Based on the analysis results, the number of samples is extracted on the basis of weight.

\section{Conclusion}

In the study of five factors affecting food safety, this paper should focus on three factors: pesticide residues, food additives and pollutants. The weight of pesticide residues is 0.5802 , which is the most important factor affecting food safety. Therefore, food safety from pesticide residues seized, hereby give the following recommendations: 
(1) The government departments should strengthen supervision of vegetable pesticide residue in food and food additives control, increase the importance of vegetables and other food pesticide scientific fertilization, and strengthen the management of drug control, raw food safety fundamentally, and formulate related standards of food additives to improve the.

(2) We strictly prohibit illegal food additives, strengthen enterprise self-discipline, establish a long-term regulatory mechanism, strictly control the product labeling of food additives, and fundamentally solve the problem of food safety of consumers.

(3) The administrative departments should strengthen the food production enterprises to standardize the education, improve the laws and regulations on food producers, food safety awareness, so that food production enterprises to understand the use of food additives, the correct understanding of the scope of use, the use of food additives, to avoid illegal food additives in food in addition.

(4) We should expand publicity, so that consumers understand the food additives, understand the role of food additives, and correctly select products containing food additives, so that consumers and sellers to achieve a win-win situation.

(5) We should actively promote green or sustainable production technologies to reduce dependence on agriculture and livestock supported by chemicals.

\section{References}

[1] Zhu Feng, Li Weike. The evaluation of lead and cadmium contamination in vegetable soldin Nanshan district by food safety indexes method[J]. Chinese Journal of Health Laboratory Technology, 2015, 25(2): 274-276.

[2] Wang Huimin,Qiao Juan. Analyze on Farmers’ Behavior and Produce Efficiency by Participating in Food Safety Traceability System: CaseofVegetableFarmersinBeijing [J].Issues in Agricultural Economy, 2011(2): 45-51+111.

[3] Cao Na, Zhang Xinyi, Jiang Li, Aminah, Yu Zhifang. Safety evaluation of fresh-cut vegetables during marketing period [J].Science and Technology of Food Industry, 2016, 37(13): 314-318+322.

[4] Zhou Jiehong, Li Kai, Liang Qiao. Food safety controls in different governance structures in China's vegetable and fruit industry [J]. Journal of Integrative Agriculture, 2015, 14(11): 2189-2202. 